\title{
FLOWRATE MEASUREMENT ON METAL PIPES BY AIR-COUPLED ULTRASOUND
}

\author{
KEISUKE TSUKADA ${ }^{1} \&$ HIROSHIGE KIKURA ${ }^{2}$ \\ ${ }^{1}$ Graduate School of Science and Engineering, Tokyo Institute of Technology. \\ ${ }^{2}$ Laboratory for Advanced Nuclear Energy, Tokyo Institute of Technology.
}

\begin{abstract}
The characteristics of the measurement capacity of air-coupled ultrasonic sensor influenced by the incident angle were investigated analytically and experimentally. The optimized incident angle between ultrasound and test pipe was determined. The air-coupled ultrasonic sensor with this determined incident angle was applied to the flowrate measurement in the aluminium pipe. The measurement results were compared to those obtained by using the electromagnetic flowmeter. Since the measurement results show good linearity, the capacity of the air-coupled ultrasonic flowmeter is revealed.

Keywords: air-coupled ultrasonic flowmeter, incident angle, lamb wave, non-contact measurement.
\end{abstract}

\section{INTRODUCTION}

Monitoring and controlling the working condition by flowmeters can contribute to the processing efficiency of the industrial facilities. Ultrasonic flowmeter [1,2] has an advantage of non-invasive measurement and more easily-setup for the measurement apparatus than the conventional flowmeters such as pressure difference flowmeter and orifice flowmeter. Additionally, the ultrasonic flowmeter can be applied to the high-accuracy measurement because of the acoustic technical development and the advanced signal processing [3, 4]. However, the ultrasonic flowmeter has a limitation of the working temperature. The piezo-electric element in the ultrasonic sensor loses sensitivity in the high-temperature condition in the thermal power plants or nuclear power plants. Thus, the conventional ultrasonic flowmeter is not applicable to the high-temperature environments. In order to overcome the problem, the air-coupled ultrasound is applied to the ultrasonic flowmeter for insulting the high-temperature condition. The air-coupled ultrasound is a sound that propagates in the air and enters into the test media. In this method, sensors are not required to contact with the test media. For instance, the air-coupled ultrasound has been developed in the non-destructive inspection (NDI) field [5, 6]. Particularly, it was adopted to the non-contact NDI to detect the cracks in ceramic materials at high-temperature condition [7]. In our study, the air-coupled ultrasonic flowmeter is developed for the high-temperature fluids. In the air-coupled ultrasonic flowrate measurement, the amplitude of received signals decreases, because of the reflection between the air and the test media. The reflection causes the acoustic loss. Especially, the acoustic loss can be estimated as roughly $100 \mathrm{~dB}$ in the metal pipe application, because of mismatching between the acoustic impedances of air and metal pipes. The acoustic loss depends on the incident angle, frequency of ultrasound and the thickness of the pipe wall [8]. In this study, the characteristics of acoustic loss influenced by the incident angle were investigated analytically and experimentally. The optimized incident angle was determined firstly, and then the air-coupled ultrasonic sensor with this determined incident angle was applied to the flowrate measurement in the aluminum pipe. 


\section{THE MEASUREMENT PRINCIPLE OF ULTRASONIC FLOWMETER}

\subsection{The measurement principle of the conventional ultrasonic flowmeter}

In the conventional ultrasonic flowmeter, two ultrasonic sensors are contacted with the pipe, and the ultrasound is transmitted from one sensor to the flow and propagated in the fluid. The ultrasound propagating in fluid is affected by flow velocity. Therefore, the time of flight (TOF) is decreased when the ultrasound propagates from upstream to downstream. By contrast, the TOF increases when the ultrasound propagates from downstream to upstream. The TOF is linear to the average flowrate in the pipe. Thus, the flowrate can be calculated from the given TOF.

\subsection{The measurement principle of the air-coupled ultrasonic flowmeter}

The air-coupled ultrasound generated by the flowmeter emits from air to the pipe. Ultrasonic waves are refracted at the interface between substances as shown in Fig. 1. Conventional ultrasonic flowmeters have acoustic couplant between the sensors and the pipe. The sound velocity in the couplant materials is almost equal to that in water. Accordingly, the refracted angle becomes almost equal to the incident angle. However, the angle of refraction in air-coupled ultrasonic flowmeters is not equal to the angle of incidence. The setup of air-coupled ultrasonic sensors is shown in Fig. 2. The TOF difference between two ultrasonic transmissions in the upstream and downstream of the flow through the same path line has a relationship with the fluid flow rate.

The incident angle $\theta$ and the refracted angle $\phi$ is related to the sound velocity in the water $\mathrm{C}_{\mathrm{w}}$ and that in the air $\mathrm{C}_{\mathrm{a}}$ when wave propagates from the air to water. The relationship is denoted as

$$
\frac{\sin \theta}{\sin \phi}=\frac{\mathrm{C}_{\mathrm{a}}}{\mathrm{C}_{\mathrm{w}}} .
$$

The TOF for the upstream of the flow $t_{\text {up }}$ is calculated as eqn (2) by using the average velocity $V$ of flow.

$$
t_{u p}=\frac{L_{1}+L_{2}}{\mathrm{C}_{\mathrm{a}}}+\frac{D}{\cos \phi\left(\mathrm{C}_{\mathrm{w}}-V \sin \phi\right)},
$$

where, $L_{1}$ and $L_{2}$ denote the distances between the surface of the pipe and the surface of ultrasonic sensors, respectively. $D$ is the diameter of the pipe. The TOF for the downstream flow $t_{\text {down }}$ is:

$$
t_{\text {down }}=\frac{L_{1}+L_{2}}{\mathrm{C}_{\mathrm{a}}}+\frac{D}{\cos \phi\left(\mathrm{C}_{\mathrm{w}}+V \sin \phi\right)} .
$$

The time difference $\Delta \mathrm{t}$ between upstream and downstream is:

$$
\Delta t=t_{\text {down }}-t_{\text {up }}=\frac{2 \mathrm{C}_{\mathrm{w}} V \tan \phi}{\mathrm{C}_{\mathrm{w}}^{2}+V \sin ^{2} \phi} .
$$

The flow velocity is much smaller than the speed of sound in water. The term of sin squared is negligible. The equation of the air-coupled ultrasonic flow meter can be simply expressed as

$$
V=\frac{\mathrm{C}_{\mathrm{w}} \Delta t}{2 \tan \phi} \text {. }
$$




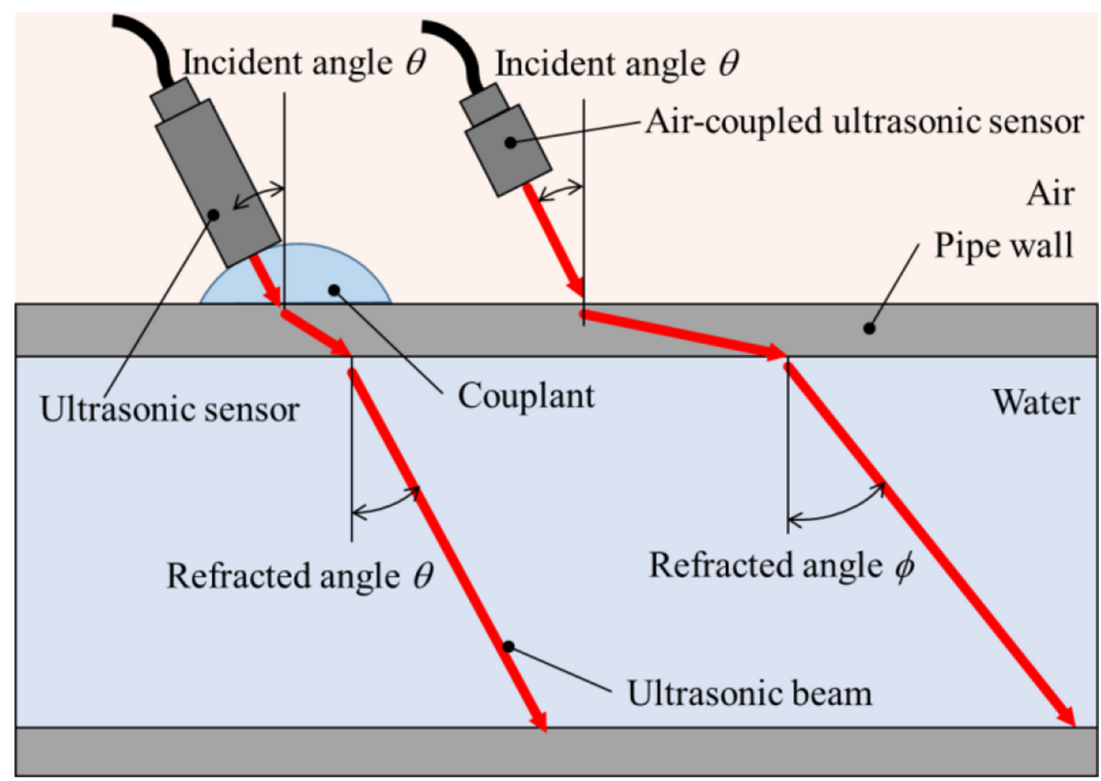

Figure 1: The refraction on the boundary between the air and the pipe.

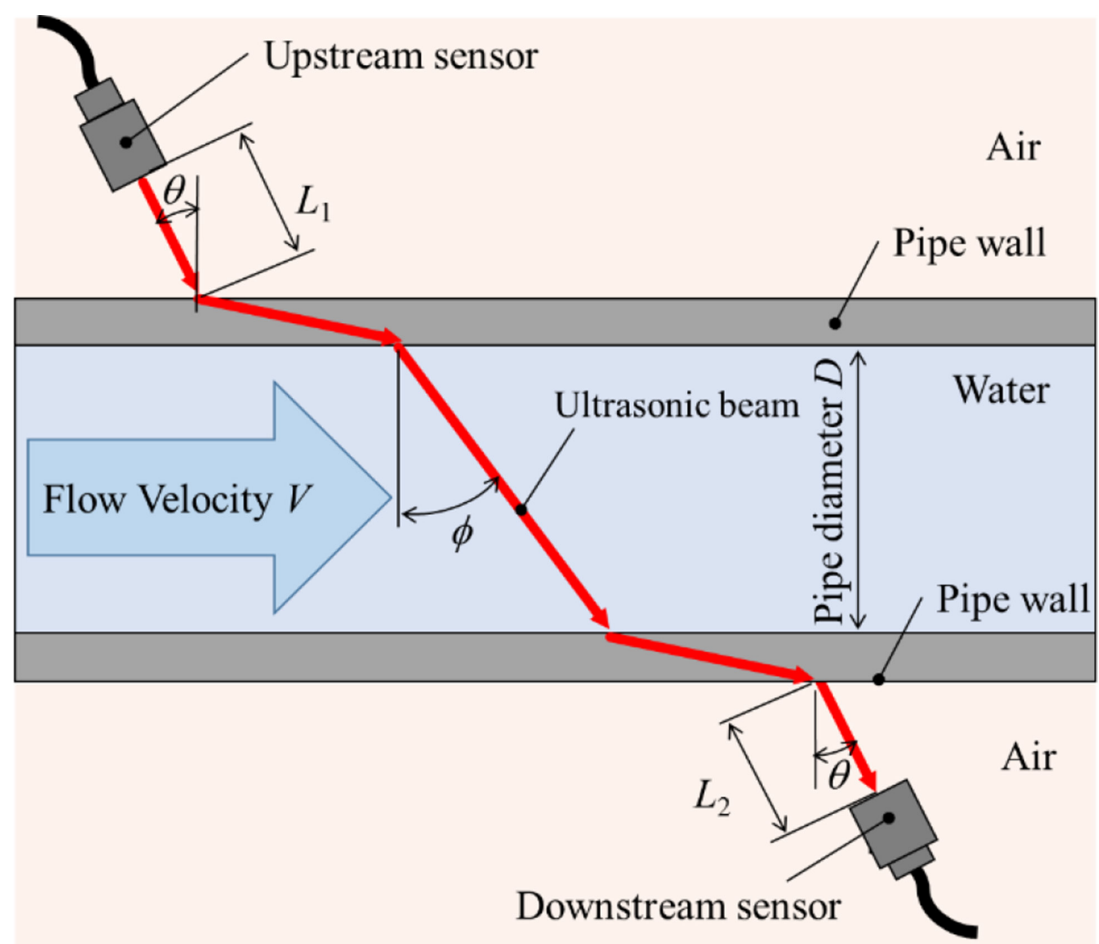

Figure 2: The setup of sensors for the air-coupled ultrasonic flowmeter. 
Then, the flowrate $Q$ can be calculated from the average velocity of fluid

$$
Q=\frac{\pi D^{2}}{4} V=\frac{\pi D^{2} \mathrm{C}_{\mathrm{w}} \Delta t}{8 \tan \phi}
$$

In order to measure the average flow velocity, the sound velocity in water, the sound velocity in air, the incident angle and time difference are required from eqns (1) and (5). The sound velocity in water and that in air can be obtained by physical properties, and the incident angle is determined by setup of sensors. Therefore, the flow rate can be simply calculated by measuring the TOF.

\section{ACOUSTIC TRANSMISSION ON THE ALUMINIUM PIPE}

\subsection{Acoustic transmission coefficient}

The air-coupled ultrasound propagates through the pipe wall during the measurement. Most of the acoustic loss happens in the boundary between pipe and air. Minimizing the acoustic loss of the boundary is one of the solutions to improve the signal to noise ratio in air-coupled ultrasonic flowmeter. The acoustic loss of bulk wave through the pipe wall depends on the acoustic impedances of air and pipe material. Moreover, the acoustic loss of the ultrasound also depends on the thickness of plate when the ultrasound passes through the plate with diagonal incident angle, because of the lamb wave. Lamb wave penetration has lower acoustic loss than bulk wave penetration, and it enables efficient transmittance through the pipe wall. Ultrasound penetrated into plate is refracted, and elects bulk wave and lamb wave. The refracted angle, ruled by Snell law, depends on the longitudinal wave and transverse wave of the sound in the plate as shown in Fig. 3. The transmission coefficient can be evaluated from acoustic impedance of media. In particular, the acoustic transmission coefficient through the thin plate was investigated by Y. Torikai [9]. In his research, ultrasonic transmission coefficient $T$ is shown in eqn (7) in case of two different medias divided by plate.

$$
T=\left|\frac{2 N \frac{v_{0}}{v_{2}} e^{i r_{2} d}}{M\left(\frac{\rho_{2} v_{2}}{\rho_{0} v_{0}}+\frac{\cos \theta_{2}}{\cos \theta_{0}}\right)+i\left[\left(N^{2}-M^{2}\right) \frac{\cos \theta_{2}}{\cos \theta_{0}}+\frac{\rho_{2} v_{2}}{\rho_{0} v_{0}}\right]}\right|^{2} .
$$

where

$$
\begin{gathered}
N=\frac{\cos \theta_{0}}{\rho_{0} v_{0}}(F+G), \quad M=\frac{\cos \theta_{0}}{\rho_{0} v_{0}}\left(F \cos r_{1} d+G \cos s_{1} d\right), \\
F=\frac{\rho_{1} v_{l} \cos ^{2} 2 \theta_{t}}{\cos \theta_{l} \sin s_{1} d}, \quad G=\frac{\rho_{1} v_{t} \sin ^{2} 2 \theta_{t}}{\cos \theta_{t} \sin s_{1} d}, \\
r_{1}=\frac{\omega}{v_{l}} \cos \theta_{l}, \quad s_{1}=\frac{\omega}{v_{t}} \cos \theta_{t}, \\
r_{2}=\frac{\omega}{v_{2}} \cos \theta_{2} .
\end{gathered}
$$




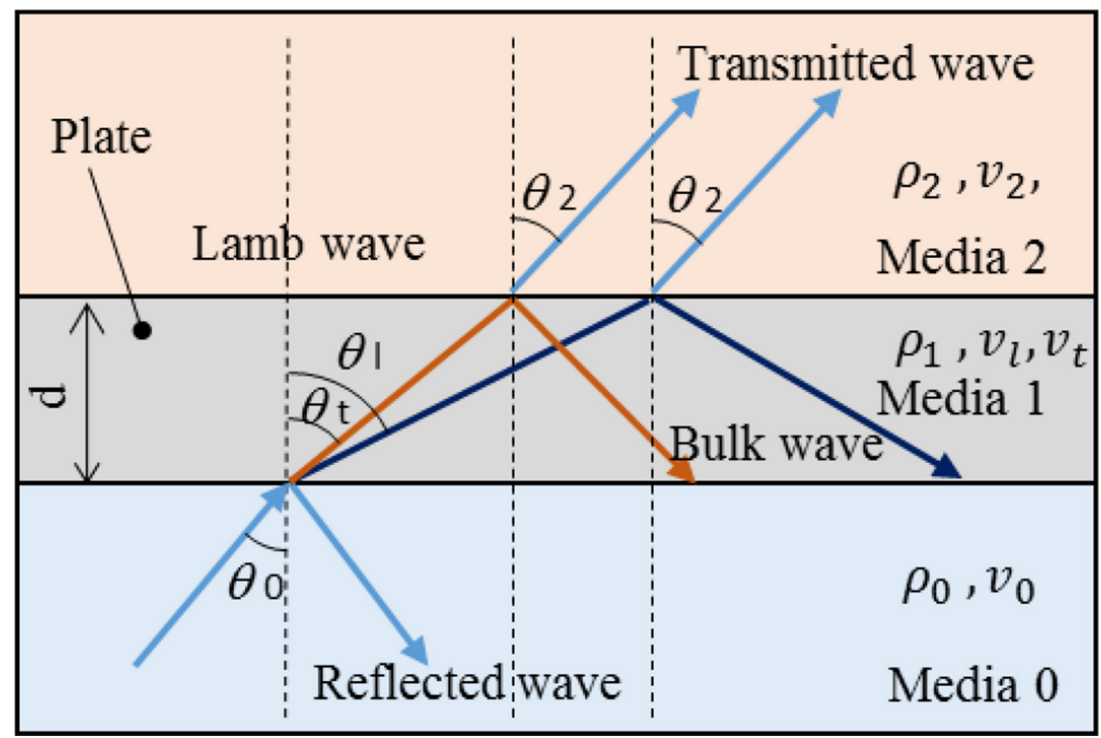

Figure 3: Analytical plane layered model for ultrasonic refraction in the plate.

Table 1: Physical properties used for acoustic transmission coefficient calculation.

\begin{tabular}{llll}
\hline & Density $\left[\mathrm{kg} / \mathrm{m}^{3}\right]$ & $\begin{array}{l}\text { Longitudinal wave } \\
\text { velocity }[\mathrm{m} / \mathrm{s}]\end{array}$ & $\begin{array}{l}\text { Transverse wave } \\
\text { velocity }[\mathrm{m} / \mathrm{s}]\end{array}$ \\
\hline Air & 1.23 & 330 & - \\
Water & 1,000 & 1,500 & - \\
Aluminium & 2,700 & 6,400 & 3,040 \\
\hline
\end{tabular}

And, $v_{0}, v_{l}, v_{t}, v_{2}, \rho_{0}, \rho_{1}, \rho_{2}$ and $\omega$ are sound velocity of media 0 , longitudinal velocity of media 1 , transverse velocity of media 1 , sound velocity of media 2 , the density of media and angle velocity of ultrasound, respectively. Table 1 shows the detailed properties of testing materials. Equation (7) is a function of incident angle $\theta_{0}$, thickness of plate $d$ and ultrasonic frequency $f$ after substituting all the given physical properties.

The acoustic transmission coefficient through the aluminum plate in water is depicted in Fig. 4. The vertical axis denotes the value of the ultrasonic frequency multiplied with the thickness of plate. The horizontal axis denotes the incident angle. Color bar shows the acoustic loss. It is found that the acoustic loss of ultrasound through aluminum can be minimized to at least $10 \mathrm{~dB}$. This is lower than that of vertical ultrasonic transmission. This result has good agreement with the result approached by same method [10]. Therefore, the code validation is verified. Figure 5 shows the acoustic transmission through aluminum plate from air to water. The acoustic transmission is maximized when the incident angle is roughly $7^{\circ}$. Acoustic loss can be minimized to at least $20 \mathrm{~dB}$. The acoustic loss with vertical transmission is approximately $40 \mathrm{~dB}$. Therefore, the transmission of ultrasound with incident angle has advantages on the improvement of acoustic transmission. And the acoustic loss is maximized 


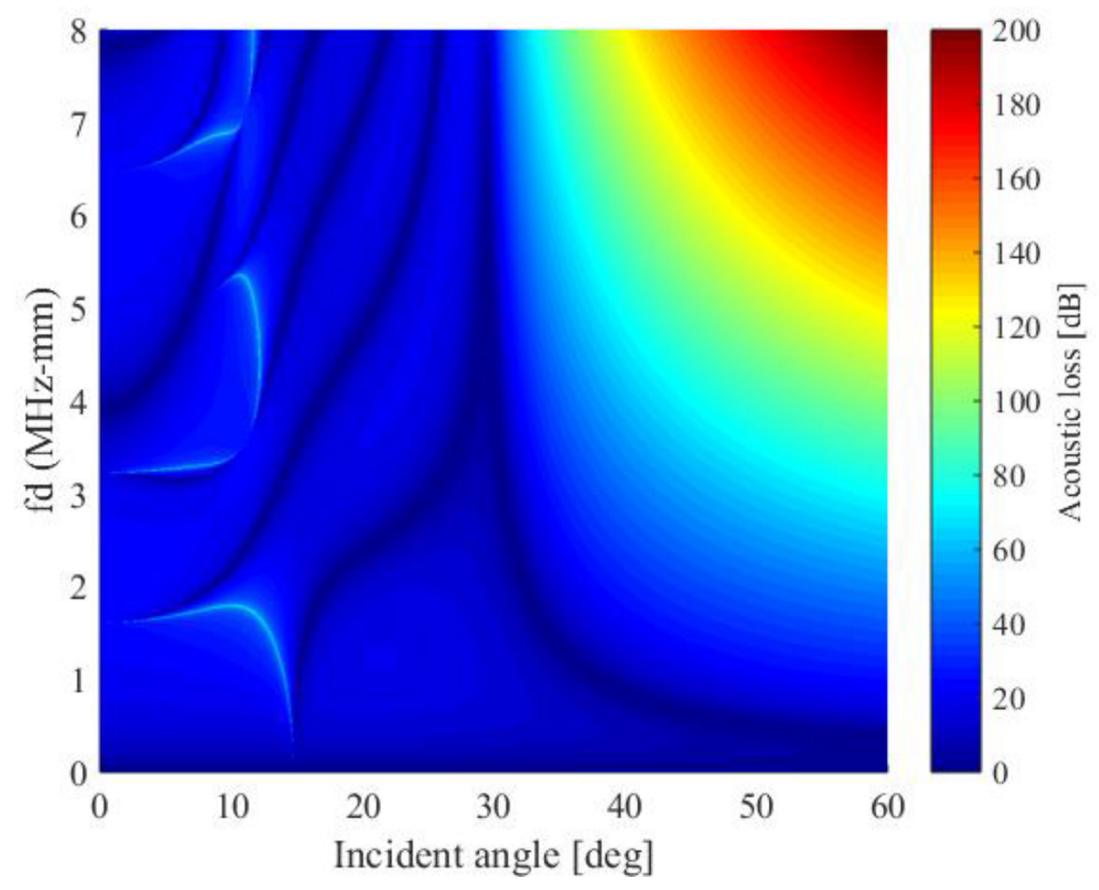

Figure 4: Acoustic transmission through aluminium plate in water.

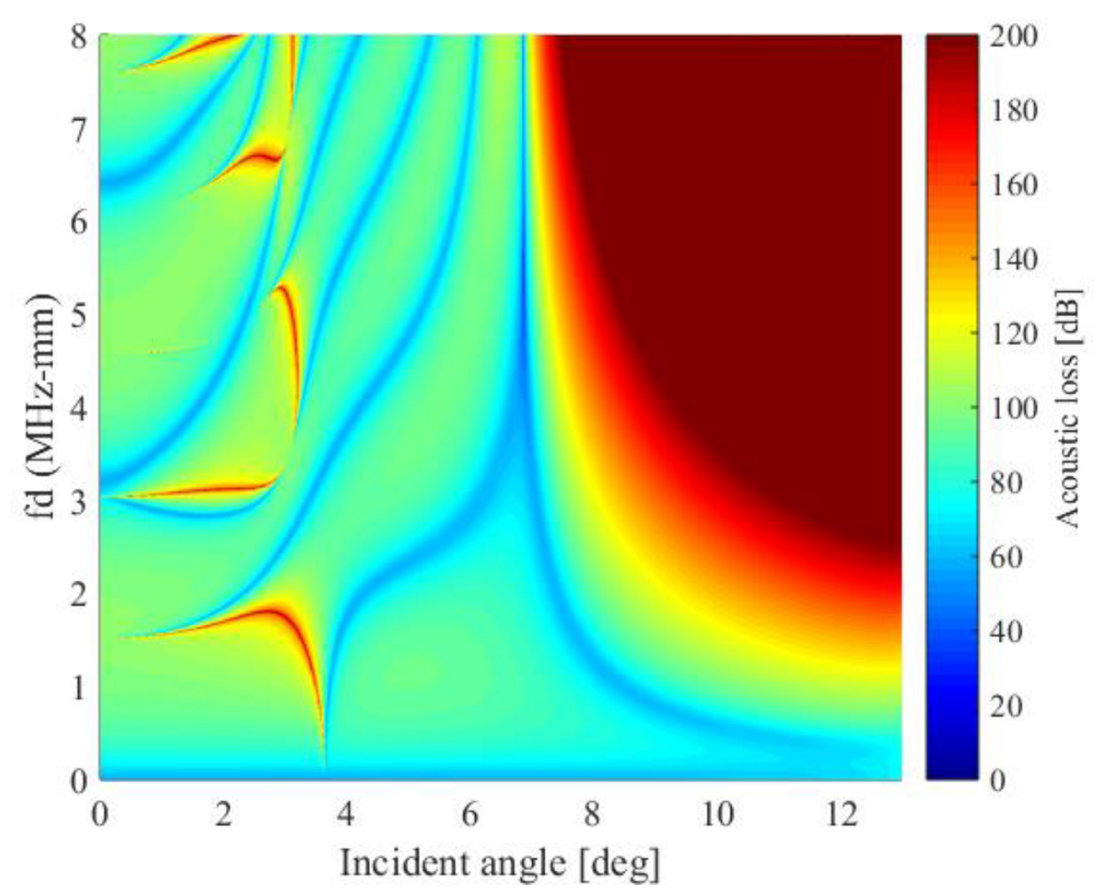

Figure 5: Acoustic transmission through aluminium plate from air to water. 
when incident angle is around $3^{\circ}$. The acoustic loss is $160 \mathrm{~dB}$ in this incident angle, and it is $120 \mathrm{~dB}$ higher than that with vertical transmission. Thus, it is properly that the acoustic loss can be minimized by incident angle by $20 \mathrm{~dB}$. However, the acoustic loss increased in certain incident angle, e.g. $3^{\circ}$. The optimized incident angle can be predicted through the calculation of the transmission coefficient.

\subsection{Experiment for the acoustic transmission}

The acoustic transmittance on aluminum pipe was investigated experimentally. The aluminum pipe with inner diameter of $56 \mathrm{~mm}$ and outer diameter of $60 \mathrm{~mm}$ was filled with water, and two ultrasonic sensors (Japan probe Co. Ltd, $0.4 \mathrm{~K} 20 \times 20 \mathrm{~N} 40 \mathrm{R}$ ) were set up as opposed between the pipe. The center frequency of sensors is $400 \mathrm{kHz}$, and the sensors have $20 \mathrm{~mm} \times$ $20 \mathrm{~mm}$ vibration surfaces. The distance between the vibrating surface of ultrasonic sensors and the pipe surface is $10 \mathrm{~mm}$ as shown in Fig. 6. In order to simplify the analytical model as plane layered model, a focusing sensor whose vibrating surface has suitable curvature to fit the pipe curvature was applied to the experiment. It is because the refraction at the pipe curvature is prevented by focusing sensors and the incident angle is constant in the vibrating surface along the pipe curvature.

The ultrasonic sensors were controlled by pulsar/receiver (Japan Probe Co. Ltd, JPR$10 \mathrm{CN})$. Ultrasound is transmitted from one sensor and received in the other sensor. The received signal was digitized by $\mathrm{AD}$ converter in the pulsar/receiver and was transported to

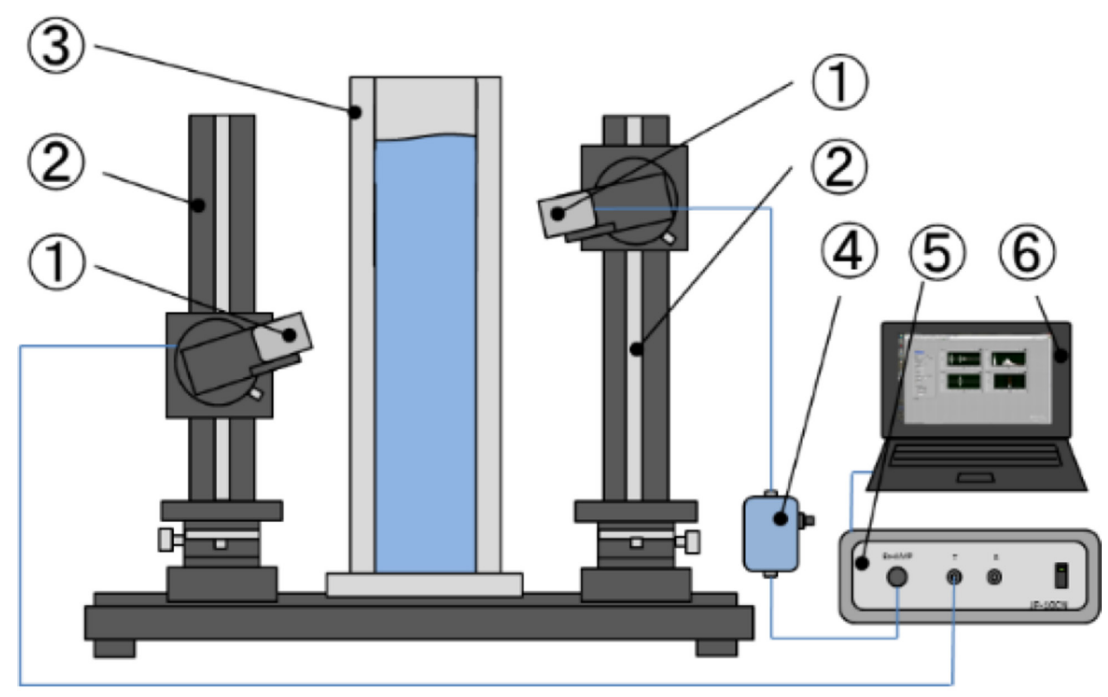

1. Ultrasonic sensors 2. Stages

3. Aluminium pipe 4. Ex-AMP

5. Pulser/Receiver 6. Personal computer

Figure 6: The experimental apparatus for optimizing the incident angle of ultrasound to the aluminum pipe. 
the personal computer. From the received signal, the acoustic intensity was evaluated. The acoustic intensity is defined as

the peak to peak of the received signal shown in Fig. 7. The acoustic intensity of transmitted ultrasound from the pipe was measured by changing the incident angle per degree in the range of $0^{\circ}-13^{\circ}$. Obtained acoustic transmittance is shown in Fig. 8. The horizontal axis denotes the incident angle to the pipe wall, and vertical axis denotes the normalized acoustic intensity. The solid line corresponds to the analytical acoustic intensity calculated in previous section.

The acoustic intensity increased at $4^{\circ}$. From the analytical results, the singular point is at $4^{\circ}$ and the first peak of acoustic transmission is caused by singular point. The acoustic transmission became lower with higher incident angle in the experiment. It is because the focusing

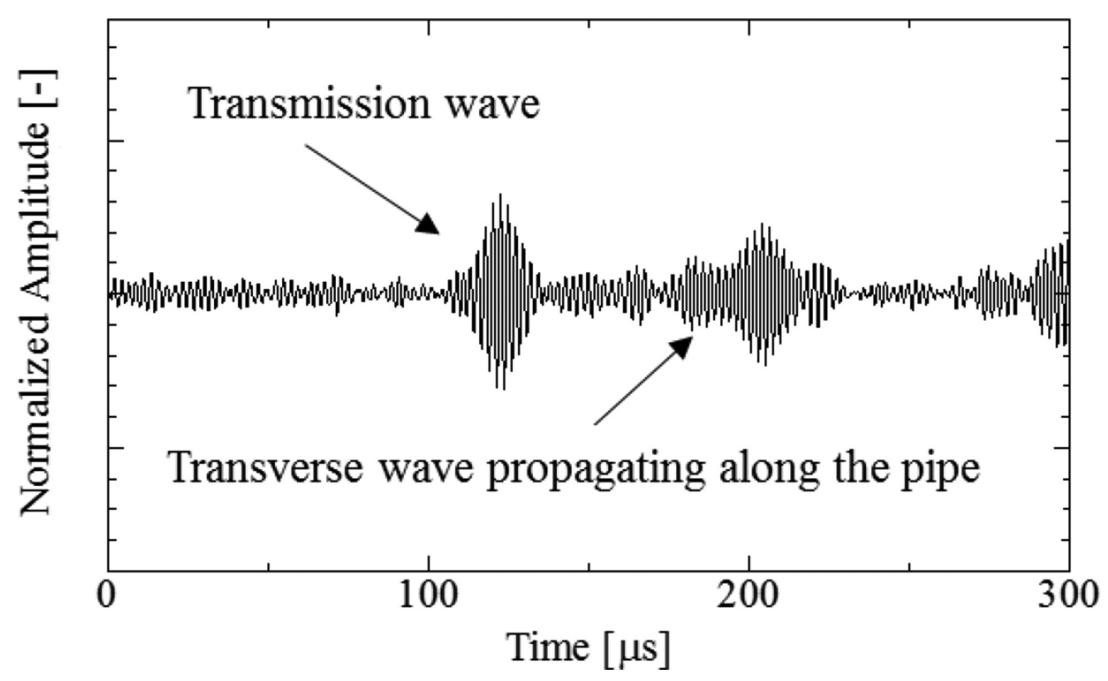

Figure 7: Detected signal in the receiving sensor with $0^{\circ}$ incident angle.

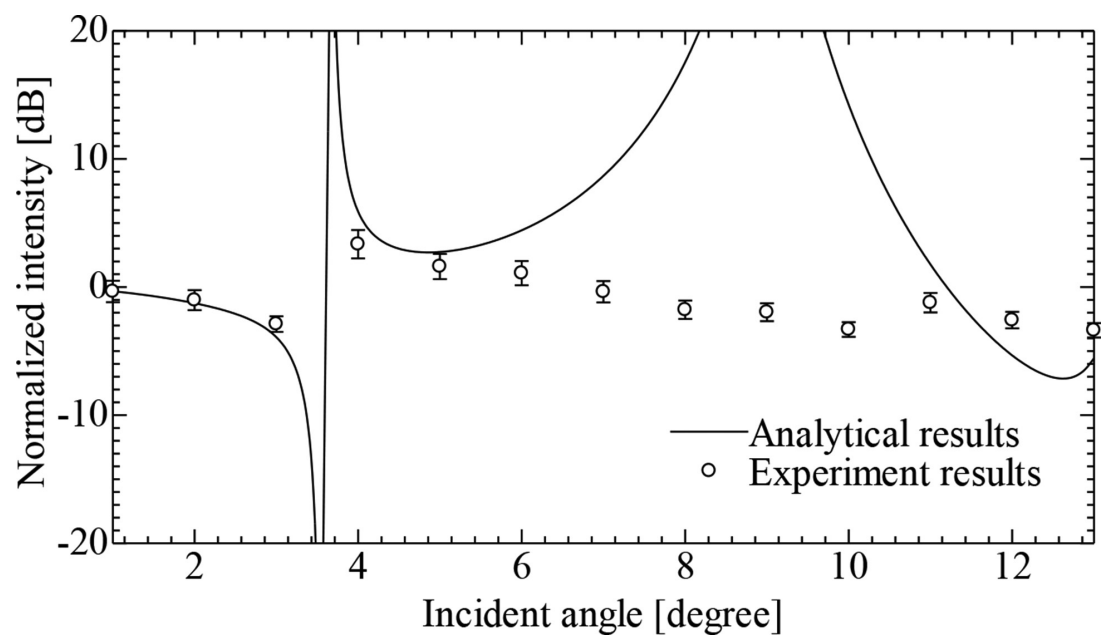

Figure 8: Acoustic transmittance on the aluminium pipe when ultrasound transits diagonally (plots shows the experiment results and solid line shows the analytical estimation). 
sensor with higher angle is not well-fit to the curvature of the pipe. This is not regarded as the plane layered model. Additionally, the intensity in the second singular point in the analytical estimation is higher than that of the experiment. As a result, the analytical estimation of acoustic transmission is valid with small incident angle up to $5^{\circ}$. For the air-coupled ultrasonic flow measurement in aluminum pipe, $4^{\circ}$ for the incident angle has maximum value of acoustic transmission.

\section{FLOWRATE MEASUREMENT}

\subsection{Experimental setup}

The flowrate measurement was performed in the aluminum pipe by applying the focusing sensor with optimized incident angle. The experimental setup is shown in Fig. 9. This apparatus consists of an electromagnetic flowmeter, a test section, and an air coupled ultrasonic flowmeter. Working fluid is tap water which is stored in the reserve tank and was pumped to the straightener by the electromagnetic flowmeter. It flows through the test section and, goes back to the reserve tank. The test section is made of aluminum pipe. The outer diameter of the aluminum pipe is $60 \mathrm{~mm}$, and the inner diameter is $56 \mathrm{~mm}$, and the length of the test section is $1000 \mathrm{~mm}$. The air-coupled ultrasonic flowmeter is set up at $2500 \mathrm{~mm}$ from the outlet of the straightener. The air-couple ultrasonic flowmeter system consists of a Pulsar/Receiver (Japan Probe Co. Ltd, JPR-10CN), an 8-bit Digitizer (National Instruments Corp., PXI-5114) which the sampling speed is $250 \mathrm{MS} / \mathrm{s}$, a PXI-Express Chassis (National Instruments Corp., PXIe1062Q) that contains a personal computer, an Ext-Amplifier. The electromagnetic flowmeter is set up at the inlet of the straightener because it has no interfere with the ultrasonic flowmeter. The electromagnetic flowmeter has $3 \%$ error of full-scale with the calibration.

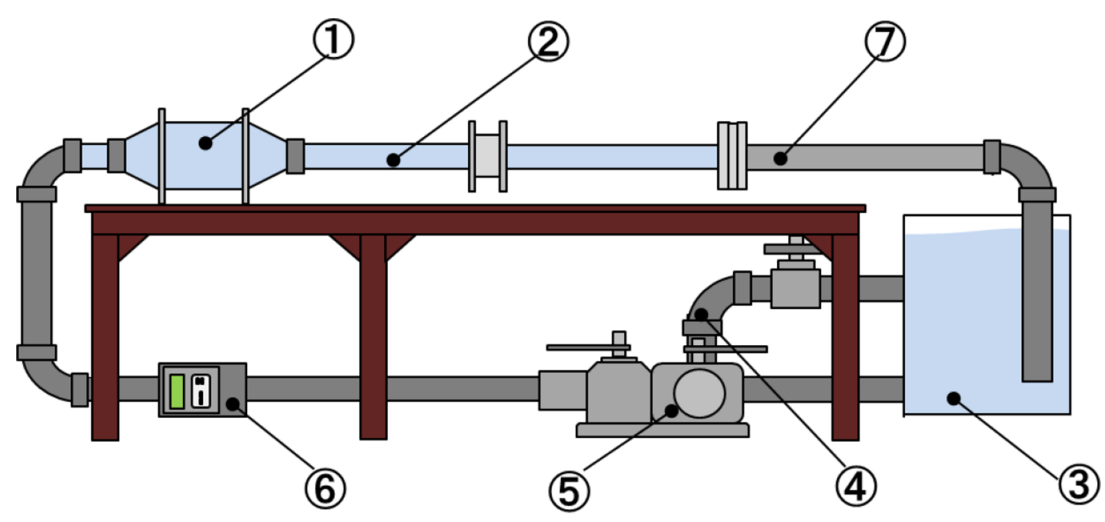
1. Straightener
2. Acrylic pipe
3. Reserve tank
4. Bypath
5. Water pump
6. Electromagnetic flowmeter
7. Test section

Figure 9: Experimental apparatus of the aluminium pipe flow rate measurement by the aircoupled ultrasonic flowmeter with optimized incident angle. 


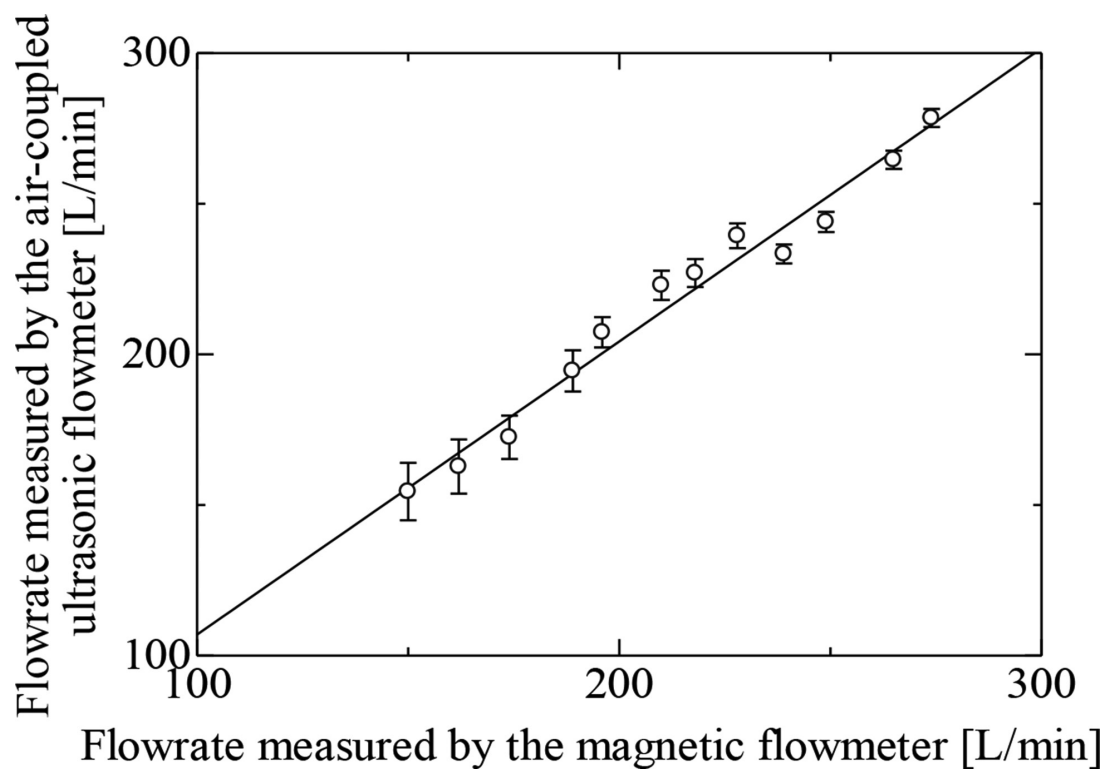

Figure 10: The result of flow rate measurements by the air-coupled ultrasonic flowmeter and the electromagnetic flowmeter.

\subsection{Flowrate measurement results}

The measured average flow rates by the air-coupled ultrasonic flowmeter compared to that by the electromagnetic flowmeter are shown in Fig. 10. The vertical axis denotes the flow rates measured by the air-coupled ultrasonic flowmeter, and the horizontal axis denotes the flow rates measured by the electromagnetic flowmeter. The measurement by the air-coupled ultrasonic flowmeter was repeated 1000 times. The plots show the average of measured flowrates. Error bar shows the standard deviations of measured flow rates. The solid line is drawn by using the least-squares method. The slope of the line is 0.973 and the coefficient of determination $\mathrm{R}^{2}$ value is 0.987 . The results show good linearity. However, the standard deviations are larger when the flowrates are low. Because in the signal processing, due to the sampling speed it is too small to detect the TOF difference within the limitation of time resolution. Thus, the capability of air-coupled ultrasonic flowmeter for the metal pipe application is revealed.

\section{CONCLUSION}

The air-coupled ultrasonic flowmeter was applied to flow rate measurement on the aluminum pipe in this study. It is known that the acoustic loss between air and aluminum is $40 \mathrm{~dB}$ higher than that between water and aluminum. In order to overcome this acoustic loss, the incident angle for air-coupled ultrasonic sensors was optimized analytically, firstly. The diagonal ultrasound transition causes refracted longitudinal wave and refracted transverse wave. In this study, the transverse wave was taken into consideration and the analytical model was plane layered model. It is revealed that low acoustic loss area exists, and this area depends on the ultrasonic frequency and thickness of the plane according to the analytical calculation. Then, in order to validate the analytical estimation, the acoustic loss was measured by 
experiment. In the experiment, ultrasonic focusing sensors were applied for reduction of refraction by pipe curvature. As a result, the experiment results are in good agreement with the analytical estimation in the small incident angle up to $5^{\circ}$. However, the acoustic loss in the experiment is higher than that in the analytical estimation in the incident angle larger than $5^{\circ}$. It is because the focusing sensors are effective only in small incident angles, and the refraction by the pipe curvature causes much acoustic loss in large incident angle. In particular, the minimum acoustic loss is obtained when the incident angle is $4^{\circ}$. Thus, the incident angle is determined as $4^{\circ}$ from the analytical estimation and the experiment.

Finally, the water flowrate measurement in the aluminum pipe was demonstrated by using the air-coupled ultrasonic flowmeter with the optimized incident angle and the electromagnetic flowmeter. The measurement results show good linearity. As a consequence, the measurement capability by air-coupled ultrasonic flowmeter for the metal pipe is revealed.

\section{REFERENCES}

[1] Baker, R.C., Flow Measurement Handbook, Cambridge University Press, pp. 312-356, 2000. http://dx.doi.org/10.1017/CBO9780511471100.015

[2] Miller, R.W., Flow Measurement Engineering Handbook, McGraw-Hill, 1989.

[3] Furuichi, N., Terao, Y. \& Takamoto, M., A calibration of a feed water flowmeter installed to model loop of a nuclear power plant using high reynolds number facility. Proceedings of 16th International Conference on Nuclear Engineering, pp. 145-151, 2008.

[4] Woodcock, J.P., Development of the ultrasonic flowmeter. Ultrasound in Medicine \& Biology, 2(1), pp. 11-18, 1975. http://dx.doi.org/10.1016/0301-5629(75)90036-8

[5] Takamoto, M., Ishikawa, H., Shimizu, K., Monji, H. \& Matsui, G., New measurement method for very low liquid flow rates using ultrasound. Flow Measurement and Instrumentation, 12(4), pp. 267-273, 2001. http://dx.doi.org/10.1016/S0955-5986(01)00023-1

[6] Solodov, I., Pfleiderer, K., Gerhard, H., Predak, S. \& Busse, G., New opportunities for NDE with air-coupled ultrasound. NDT \& E International, 39(3), pp. 176-183, 2006. http://dx.doi.org/10.1016/j.ndteint.2005.07.002

[7] Bashford, A.G., Air-coupled ultrasonic transducers for measurement of green-state ceramics at elevated temperatures. IEE Proceedings: Science, Measurement and Technology, 145(5), pp. 237-243, 1998.

http://dx.doi.org/10.1049/ip-smt:19982211

[8] Thomson, W.T., Transmission of elastic waves through a stratified solid medium. Journal of Applied Physics, 21, pp. 89-93, 1950.

http://dx.doi.org/10.1063/1.1699629

[9] Torikai, Y. \& Fusao, F., Nihon-onkyogakkai-shi, 8(1), pp. 21-27, 1954.

[10] Nishino, H., Masuda, S., Yoshida, K., Takahashi, M., Hoshino, H. \& Ogura, Y., et al., Theoretical and experimental investigations of transmission coefficients of longitudinal waves through metal plates immersed in air for uses of air coupled ultrasounds. Materials Transactions, 49(12), pp. 2861-2867, 2008.

http://dx.doi.org/10.2320/matertrans.MRA2008270 